Daniela Romero Moreno'

Lorena Gallardo-Peralta2,*

Alicia Moreno Ochoa

1. Centro de Formación Técnica. Universidad de Tarapacá. Arica. Chile.

2. Facultad de Ciencias Sociales y Jurídicas. Universidad de Tarapacá. Arica. Chile.

*Autor para correspondencia.

Correo electrónico: Igallardo@uta.cl (Lorena Gallardo Peralta).

Recibido el 4 de diciembre de 2018; aceptado el 11 de marzo de 2019.

\section{Calidad de vida relacionada con salud en personas mayores que participan activamente en agrupaciones sociales}

\author{
Health-Related Quality of Life \\ among elderly people who actively \\ participate in social groups
}

\section{RESUMEN}

Objetivos: Analizar la asociación entre calidad de vida relacionada con la salud (CVRS) y variables sociodemográficas, enfermedades crónicas y síndromes geriátricos en una muestra de personas mayores chilenas. Material y métodos: Estudio transversal de 310 personas mayores de 60 años. Se aplicó el SF-36 y se evaluó el diagnóstico de enfermedades crónicas y síndromes geriátricos. Se realizan análisis descriptivos para los diversos dominios de SF-36, y a través de modelos de regresión jerárquica se determinan las variables que se asocian a componente sumario físico, componente de sumario mental y la escala general de SF-36. Resultados: Las variables predictivas son: sexo, artrosis, trastorno de desplazamiento, incontinencia urinaria y depresión. Específicamente, la depresión es la variable más significativa en los diversos modelos contrastados. Conclusión: La participación social activa influye positivamente en la CVRS. Desde el ámbito de la atención primaria se debe seguir avanzando en protocolos de intervención en salud mental.

PALABRAS CLAVE: Calidad de vida relacionada con salud, participación social, depresión.

\section{ABSTRACT}

Objectives: To analyze the association between health-related quality of life (HR-QoL) and sociodemographic variables, chronic diseases and geriatric syndromes in a sample of Chilean elderly people. Material and methods: A cross-sectional study of 310 people over 60 years of age. The SF-36 was applied, the diagnosis of chronic diseases and geriatric syndromes was evaluated. Descriptive analyzes are carried out for the different domains of SF-36 and through hierarchical regression models the variables that are associated to physical summary component, mental summary component and the general scale of SF-36 are determined. Results: The predictive variables are: sex, arthritis, displacement disorder, urinary incontinence and depression. Specifically, depression is the most significant variable in the different contrasted models.

Conclusion: Active social participation positively influences HR-QoL. In the area of primary care, progress must continue in intervention protocols in mental health.

KEYWORDS: Health-related quality of life; social participation; depression.

\section{- INTRODUCCIÓN}

Chile es uno de los países más envejecidos de América Latina y en la actualidad el 19,3\% de su población tiene más de 60 años ${ }^{1}$. El envejecimiento es un proceso heterogéneo y determinado por diversos factores ${ }^{2}$. Particularmente, la participación social se ha transformado en uno de los pilares centrales en la promoción de un buen envejecimiento ${ }^{3}$. La participación social tiene una incidencia directa en el bienestar, ya que permite al individuo expresar su identidad, desarrollar sus habilidades y sentir que comparte con otros un propósito común ${ }^{4}$. La evidencia empírica en personas mayores chilenas confirma que la participación social tiene una influencia positiva en la calidad de vida ${ }^{5}$ en el proceso de envejecer con éxito ${ }^{6}$.

Escasos estudios han abordado la calidad de vida relacionada con salud (CVRS) en personas mayores chilenas que tienen una participación activa en organizaciones sociales. De esta manera, esta investigación tiene como objetivo analizar la asociación entre CVRS y variables sociodemográficas, enfermedades crónicas y síndromes geriátricos en una muestra de personas mayores chilenas con una participación activa en organizaciones sociales.

\section{- MATERIAL Y MÉTODO}

\section{Participantes}

Se obtuvo una muestra de 310 personas mayores con una participación activa en diversos tipos de agrupaciones sociales de la Municipalidad de Arica, Chile. Los criterios de inclusión fueron tener más de 60 años, participación activa en una agrupación social y con una antigüedad mínima de un mes. En este estudio, la participación activa se operacionaliza como la participación en alguna agrupación social (centro de día, 
agrupación deportiva, agrupación folclórica o agrupación recreativa) con una frecuencia igual o superior a una vez a la semana.

Las principales características de la muestra son: $84 \%$ mujeres, un $47 \%$ tiene entre 70 y 79 años, un $16 \%$ son indígenas, el $49 \%$ tiene pareja y el $50 \%$ participa en actividades deportivas. Respecto a la participación activa de las personas mayores entrevistadas, el $30 \%$ participa 1 vez a la semana, el $54 \%$ entre 2 y 3 veces a la semana, y el $16 \%$ restante 3 o más veces a la semana.

\section{Procedimiento}

Previa autorización de la Oficina Comunal de Adultos Mayores de la Municipalidad de Arica, el equipo de investigación informó de este estudio a las personas mayores que participan en los diversos talleres deportivos, funcionales y/o recreativos a través de carta informativa. Luego se coordinó un día y hora para la aplicación del cuestionario, que generalmente coincidía con el horario del taller. El Comité Ético de la Universidad de Tarapacá supervisó los aspectos éticos de esta investigación, cumpliendo con la Declaración de Helsinki de 1975.

\section{Instrumentos de medida}

Calidad de vida relacionada con salud. Se aplicó el Short Form 36 (SF-36) versión 2.0. Ha sido validado previamente en población chile$\mathrm{na}^{7}$. Alfa de Cronbach: 0,60.

Enfermedades crónicas. Se preguntó sobre el diagnóstico médico, en los últimos 6 meses, de las enfermedades crónicas: diabetes mellitus, hipertensión, dislipidemia, artrosis u otra enfermedad.

Síndromes geriátricos. Se aplicó una escala dicotómica para evaluar la existencia de síndromes geriátricos como: 1) trastornos del desplazamiento: caídas e inmovilidad; 2) incontinencia urinaria; 3) trastornos cognitivos: delirio, demencia y enfermedad de Alzheimer, y 4) depresión.

\section{Análisis de datos}

Se realizaron análisis descriptivos para los diversos dominios de SF-36. Posteriormente se contrastaron modelos de regresión lineal múltiple para el componente sumario físico (CSF), el componente sumario mental (CSM) y la escala general de SF-36. Específicamente se contrastaron dos modelos, el modelo 1 incluía variables de control $(1=$ mujer, edad, 1 = indígenas y 1 = tener pareja) y en el modelo 2 se agregan variables de enfermedades crónicas (diabetes, hipertensión, dislipidemia, artrosis u otra enfermedad crónica) y síndromes geriátricos (trastorno del desplazamiento, incontinencia urinaria, trastorno cognitivo y depresión). Los datos fueron analizados a través del programa estadístico SPSS versión 23.

\section{- RESULTADOS}

En la tabla 1 se presentan los estadísticos descriptivos de los diversos dominios de SF-36. En general, los valores son altos, especialmente para función física y social. Destaca el hecho de que no se presentan valores igual a 0 (valor mínimo que representa un peor estado de salud) para función física, vitalidad, salud mental, CSF y CSM.

Los modelos de regresión lineal múltiple para CSF, CSM y SF-36 se presentan en la tabla 2. Los resultados indican que CSF se relaciona negativamente con ser mujer, con problemas de artrosis, con trastornos de desplazamiento y con depresión. La varianza explicada para
Tabla 1. Descriptivos del cuestionario SF-36

\begin{tabular}{|l|c|c|c|c|}
\hline Dominios SF-36 & Media & DE & $\begin{array}{c}\text { Rango } \\
\text { teórico }\end{array}$ & $\begin{array}{c}\text { Rango } \\
\text { observado }\end{array}$ \\
\hline Salud general & 67,11 & 21,08 & $0-100$ & $5-100$ \\
\hline Función física & 81,6 & 18,3 & $0-100$ & $15-100$ \\
\hline Rol físico & 76,47 & 22,9 & $0-100$ & $0-100$ \\
\hline Dolor corporal & 62,47 & 25,88 & $0-100$ & $0-100$ \\
\hline Vitalidad & 69,31 & 20,37 & $0-100$ & $10-100$ \\
\hline Función social & 78,99 & 21,88 & $0-100$ & $0-100$ \\
\hline Rol emocional & 75,53 & 23,73 & $0-100$ & $0-100$ \\
\hline Salud mental & 73,72 & 18,88 & $0-100$ & $20-100$ \\
\hline $\begin{array}{l}\text { Componente sumario } \\
\text { físico }\end{array}$ & 49,89 & 7,29 & $0-100$ & $20-100$ \\
\hline $\begin{array}{l}\text { Componente sumario } \\
\text { mental }\end{array}$ & 49,75 & 9,10 & $0-100$ & $20-100$ \\
\hline
\end{tabular}

DE: desviación estándar.

CSF es del $14,3 \%$, en tanto que para el CSM, las variables predictivas son: sexo, incontinencia urinaria y depresión; todas ellas se relacionan negativamente con la variable dependiente y la varianza explicada para CSM es de $14,7 \%$. Finalmente, los modelos para SF-36 indican que las variables predictivas son sexo, tener pareja, artrosis, trastorno de desplazamiento y depresión. En suma, la CVRS se relaciona positivamente con tener pareja, pero negativamente con ser mujer, tener algún trastorno de desplazamiento y depresión; la varianza explicada para SF-36 es del 12,4\%.

\section{- DISCUSIÓN}

El objetivo de este estudio era analizar la asociación entre CVRS y variables sociodemográficas, enfermedades crónicas y síndromes geriátricos en una muestra de personas mayores chilenas con una participación activa en organizaciones sociales. Los datos nos permiten afirmar que la participación social es un factor protector en edades avanzadas, concretamente, la participación activa en organizaciones sociales tiene incidencia en la CVRS. Los valores descriptivos de los diversos dominios de SF-36 indicarían valores de regulares a buenos en la percepción de salud (medias > 60).

La depresión es la variable predictiva más significativa $(p<0,001)$ para CVRS. Al respecto, este dato es relevante y coherente si consideramos la alta incidencia de este trastorno neuropsiquiátrico en población chilena mayor de 60 años $^{8}$. Este hallazgo invita a profundizar en la influencia que tiene la participación social como recurso psicosocial que promueve el bienestar en la vejez 6 .

Asimismo, las mujeres se muestran como el grupo social más vulnerable al tener una peor percepción de salud, lo que concuerda con la evidencia empírica ${ }^{9,10}$. Por tanto, se torna necesario abordar empíricamente la incidencia de la participación social desde una perspectiva de género.

Igualmente, la artrosis es la única enfermedad crónica que se relacionaría negativamente con CVRS, lo que demostraría cómo otras enfermedades crónicas, como la diabetes, con una adecuada adhesión al tratamiento mejoraría la calidad de vida del paciente ${ }^{11}$. No obstante, los dolores y malestares de la artrosis siguen teniendo una repercusión en el bienestar de las personas mayores, especialmente es una limitación funcional. De ahí la importancia de complementar el tratamiento 
Tabla 2. Regresión jerárquica para CSF, CSM y SF-36, según variables sociodemográficas y geriátricas

\begin{tabular}{|c|c|c|c|c|c|c|c|c|c|c|}
\hline & & \multicolumn{3}{|c|}{ CSF } & \multicolumn{3}{|c|}{ CSM } & \multicolumn{3}{|c|}{ SF-36 } \\
\hline & & B & E.T. & $\beta$ & B & E.T. & $\beta$ & B & E.T. & $\beta$ \\
\hline \multirow{4}{*}{$\begin{array}{l}\text { Modelo } 1 \\
\text { Variables } \\
\text { sociodemográficas }\end{array}$} & Sexo & $-2,540$ & 1,122 & $-0,129^{b}$ & $-2,909$ & 1,414 & $-0,118^{a}$ & $-0,049$ & 0,022 & $-0,128^{b}$ \\
\hline & Edad & $-0,500$ & 1,305 & $-0,022$ & 2,275 & 1,645 & 0,081 & 0,032 & 0,026 & 0,073 \\
\hline & Etnia & $-0,873$ & 1,119 & $-0,044$ & $-0,850$ & 1,410 & $-0,034$ & $-0,018$ & 0,022 & $-0,049$ \\
\hline & Tiene pareja & 1,491 & 0,864 & 0,101 & 1,270 & 1,088 & 0,069 & 0,030 & 0,017 & $0,106 a$ \\
\hline \multirow{13}{*}{$\begin{array}{l}\text { Modelo } 2 \\
\text { Variables geriátricas }\end{array}$} & Sexo & $-1,859$ & 1,122 & $-0,095$ & $-2,562$ & 1,405 & $-0,104^{a}$ & $-0,040$ & 0,022 & $-0,106^{a}$ \\
\hline & Edad & $-0,822$ & 1,267 & $-0,037$ & 1,309 & 1,587 & 0,047 & 0,022 & 0,025 & 0,051 \\
\hline & Etnia & $-1,052$ & 1,093 & $-0,053$ & $-0,403$ & 1,369 & $-0,016$ & $-0,018$ & 0,022 & $-0,047$ \\
\hline & Tiene pareja & 0,974 & 0,835 & 0,066 & 0,574 & 1,045 & 0,031 & 0,020 & 0,016 & 0,072 \\
\hline & Diabetes & 0,934 & 1,000 & 0,052 & $-0,273$ & 1,252 & $-0,012$ & 0,022 & 0,020 & 0,063 \\
\hline & Hipertensión & $-0,453$ & 0,808 & $-0,031$ & $-0,270$ & 1,011 & $-0,015$ & 2,956 & 0,016 & 0,001 \\
\hline & Dislipidemia & 0,228 & 1,153 & 0,011 & 1,661 & 1,444 & 0,063 & 0,009 & 0,023 & 0,021 \\
\hline & Artrosis & $-3,045$ & 0,961 & $-0,183^{b}$ & $-0,878$ & 1,204 & $-0,042$ & $-0,034$ & 0,019 & $-0,107^{a}$ \\
\hline & Otra enfermedad & $-1,528$ & 1,073 & $-0,080$ & $-0,303$ & 1,343 & $-0,013$ & $-0,009$ & 0,021 & $-0,024$ \\
\hline & Tras. desplazamiento & $-5,045$ & 1,633 & $-0,175^{b}$ & $-1,605$ & 2,045 & $-0,044$ & $-0,060$ & 0,032 & $-0,107^{a}$ \\
\hline & Incontinencia urinaria & 0,178 & 1,394 & 0,007 & $-3,567$ & 1,746 & $-0,116 b$ & $-0,020$ & 0,028 & $-0,041$ \\
\hline & Trastornos cognitivos & 0,361 & 4,961 & 0,004 & 5,426 & 6,212 & 0,048 & 0,030 & 0,097 & 0,017 \\
\hline & Depresión & $-3,236$ & 1,317 & $-0,136^{c}$ & $-9,098$ & 1,650 & $-0,305^{c}$ & $-0,104$ & 0,026 & $-0,227^{c}$ \\
\hline \multicolumn{2}{|l|}{$\mathrm{R}^{2}$} & \multicolumn{3}{|c|}{0,143} & \multicolumn{3}{|c|}{0,147} & \multicolumn{3}{|c|}{0,124} \\
\hline \multicolumn{2}{|l|}{ Cambio en F } & \multicolumn{3}{|c|}{$3,736^{c}$} & \multicolumn{3}{|c|}{$3,861^{c}$} & \multicolumn{3}{|c|}{$3,153^{c}$} \\
\hline
\end{tabular}

clásico de antiinflamatorios no esteroideos con ejercicio terapéutico de fortalecimiento y la fisioterapia ${ }^{12}$.

Los síndromes geriátricos, tales como trastornos de desplazamiento e incontinencia urinaria, se asocian con peor CVRS. La evidencia empírica ha demostrado que la falta de autonomía influye negativamente en la valoración de la calidad de vida ${ }^{13}$. En Chile poco se ha profundizado sobre estrategias de integración de personas mayores con algún grado o tipo de discapacidad. En términos generales, las personas mayores chilenas que participan en agrupaciones sociales son autovalentes en términos funcionales.

Este estudio presenta algunas limitaciones que se deben tener en cuenta. En primer lugar, el diseńo transaccional invita a tener cautela en la interpretación causal de las variables. En segundo lugar, la muestra no es representativa de la población nacional; por tanto, no se pueden generalizar los hallazgos encontrados.

Concluyendo, este estudio abre espacio a seguir profundizando en la línea de investigación sobre participación social y CVRS. Los resultados de esta investigación muestran que la CVRS, en personas mayores con una participación social activa, se relaciona con variables sociodemográficas, enfermedades crónicas y síndromes geriátricos. Destaca la relevancia de la depresión como determinante del bienestar en población mayor chilena y también en otros contextos geográficos ${ }^{14}$ -

\section{Conflicto de intereses}

Los autores declaran no tener ningún conflicto de intereses.

\section{- BIBLIOGRAFÍA}

1. CASEN 2017. Encuesta de Caracterización Socioeconómica. Adultos Mayores: Síntesis de resultados. Subsecretaria de Evaluación Social. Ministerio de Desarrollo Social.

2. Kok AA, Aartsen MJ, Deeg DJ, Huisman M. Capturing the Diversity of Successful Aging: An Operational Definition Based on 16-Year Trajectories of Functioning. Gerontologist. 2017:57:240-51

3. Kim SH, Park S. A Meta-Analysis of the Correlates of Successful Aging in Older Adults. Res Aging. 2017:39:657-77.

4. Howrey BT, Hand CL. Measuring Social Participation in the Health and Retirement Study. Gerontologist. 2018. Avance en línea. https://doi.org/10.1093/geront/gny094

5. Herrera MS, Barros C, Fernández MB. Predictors of quality of life in old age: a multivariate study in Chile. J Popul Ageing. 2011:4:121-39.
6. Gallardo-Peralta L, Conde-Llanes D, Córdova-Jorquera I. Asociación entre envejecimiento exitoso y participación social en personas mayores chilenas. Gerokomos. 2016;27:104-8

7. Olivares-Tirado P. Estado de Salud de Beneficiarios del Sistema de Salud de Chile, 2004-2005. Gobierno de Chile. Superintendencia de Isapres, Departamento de Estudios y Desarrollo Santiago de Chile, Ministerio de Salud; 2007.

8. Sandoval FA, Tamiya N, Lloyd-Sherlock P, Noguchi H. Relation of depression with health behaviors and social conditions of dependent community-dwelling older persons in the Republic of Chile. Int Psychogeriatr. 2016;28:2029-43

9. Muñoz F, Espinoza JM. Envejecimiento activo y desigualdades de género. Aten Primaria. 2008:40:305-9.

10. Gallardo-Peralta L, Córdova I, Piña M, Urrutia B. Diferencias de género en salud y calidad de vida en personas mayores del norte de Chile. Polis. 2018;17:153-75.
11. Ortiz M, Ortiz E. Adherencia al tratamiento en adolescentes diabéticos tipo 1 chilenos: una aproximación psicológica. Rev. méd. Chile. 2005;133:307-13.

12. Jiménez C, Fernández R, Zurita F, Linares D, Farías A. Programas de Educación en Salud y Entrenamiento de la Fuerza en adultos mayores con artrosis de cadera leve a moderada. Rev. méd. Chile. 2014;142:436-42.

13. Fry PS. Predictors of Health-Related Quality of Life Perspectives, Self-Esteem, and Life Satisfactions of Older Adults Following Spousal Loss: An 18-Month Follow-up Study of Widows and Widowers. Gerontologist. 2001;41:787-98.

14. Naveiro-Rilo JC, Diez-Juárez D, Flores-Zurutuza ML, Javierre $\mathrm{P}$, Alberte $\mathrm{C}$, Molina R. La calidad de vida en ancianos polimedicados con multimorbilidad. Rev Esp Gerontol. 2014:49:153-98 